\title{
Der doppelte Franz und das Danaergeschenk
}

\author{
Kommentar zu Thomas Nisters
}

\section{Peter Nickl}

Angenommen: 4. März 2021 / Online publiziert: 26. April 2021

(C) Der/die Autor(en) 2021

Zusammenfassung Während Thomas Nisters eine Parallele zwischen Dankbarkeit und Ärger zu ziehen versucht, könnte die Pointe der Geschichte in einer mehrfachen Verdoppelung zu sehen sein: der schenkenden Handlung, der Gabe, der beschenkten Person, des Schenkenden. Der Umschlag von Dankbarkeit zu Ärger ist weniger verwunderlich, wenn wir uns an die Lehre von Thomas von Aquin erinnern, dass „die Vergeltung einer Gunst mehr vom affectus des Gebenden als vom effectus [der Gabe] abhängt."

Die brillanten Ausführungen von Thomas Nisters möchte ich um fünf Aspekte ergänzen.

1. Die Pointe der Schnitzler-Novelle, in der dieselbe Handlung - das Geschenk der Goldmünze an den mittellosen Studenten Franz - sowohl überschwängliche Dankbarkeit als auch gröbste Undankbarkeit auslöst, funktioniert nur dadurch, dass es ja eigentlich zu einer Verdopplung dieser Handlung kommt. Wodurch?

Nun, zunächst ist Franz einfach dankbar, weil ihm durch eine völlig unvorhersehbare Großzügigkeit aus der Not geholfen wurde: er hat Hilfe erfahren.

Da er aber mit dem Geschenk nichts Rechtes anzufangen weiß, es verprasst und verhurt, hat sich die großzügige Gabe sozusagen ex post in ein Danaergeschenk verwandelt. Es zeigt, so kann man vermuten, Franz wieder einmal seine Charakterschwäche, die ihn vielleicht auch in seine prekäre Ausgangslage gebracht hat. Für einen anderen wäre das Goldstück der Boden gewesen, auf dem sich eine neue Existenz hätte aufbauen lassen. Für Franz war es die Bestätigung der Erfahrung: mir entgleitet alles, ich habe mich nicht in der Hand. Für einen tugendhaften

P. Nickl (凷

Institut für Philosophie, Universität Regensburg, 93040 Regensburg, Deutschland

E-Mail: peter.nick1@phil.uni-hannover.de 
Empfänger wäre die Gabe das, was sie sein sollte - in der Hand des Lasterhaften verkehrt sie sich tatsächlich in ein Übel. Aber diese Entdeckung braucht Zeit, wenigstens ein paar Stunden; Stunden, in denen die Gabe ihren Charakter ins Gegenteil verkehrt.

Wir stellen also fest: Die Ambivalenz von Franz' Reaktion (erst Dankbarkeit, dann Undankbarkeit) gilt nicht paradoxerweise ein und derselben Handlung, sondern zwei verschiedenen Handlungen, sie entspricht der Ambivalenz des Geschenks, das zunächst ein Gut, aus der Retrospektive aber ein Übel ist. Anders gewendet: der Empfänger scheint zunächst einfach ein Bedürftiger zu sein, dem durch die großzügige Gabe geholfen sein müsste. Im Verlauf einiger Stunden stellt sich aber heraus, dass dieser Bedürftige zugleich charakterschwach ist, so dass sich in seinen Händen die gute Gabe keineswegs zum Guten auswirkt. Wir könnten also auch von einem ,doppelten Franz“" sprechen.

(Drei Verdoppelungen, je nach Perspektive: der schenkenden Handlung, der Gabe, der beschenkten Person.)

2. Wir haben also die den Dank (bzw. den Undank) auslösende Handlung verdoppelt und erklären daraus die doppelte Reaktion. Nisters verfährt so, dass er die doppelte Reaktion in eine vereinheitlichende Perspektive bringt: Dankbarkeit und ,granger“ (Dankbarkeits-Ärger) haben mindestens drei gemeinsame Aspekte, so dass sie einander stark angenähert werden können. Ja, formal lässt sich sogar eine Gleichheit der moralisch gegensätzlich beurteilten Haltungen konstruieren: beide verabscheuen die Ungleichheit, die zwischen Geber und Empfänger zutage getreten ist, allerdings schaffen sie Abhilfe in entgegengesetzter Richtung. Während der Dankbare (wie der Ehrgeizige) einen „Ausgleich nach oben“ anstrebt, versucht der Undankbare (wie der Neidische) einen „Ausgleich nach unten“. Jedoch lässt sich die Ungleichheit in keinem Fall vollkommen beseitigen. Dies sieht auch Thomas von Aquin so, allerdings in einer Perspektive, die Nisters nicht explizit übernimmt. Die Forderung des Ausgleichs, die von einem Geschenk ausgeht, betrifft nach Thomas nicht so sehr den ,effectus“, also die materielle Seite der Wohltat, sondern den ,affectus“, die emotionale Zuwendung zum Geber (vgl. STh II-II, q. 106, a. 5). Das wäre vielleicht das Kriterium, nach dem Nisters Ausschau hält, wenn es darum geht, die zulässigen Partikular-Asymmetrien zu operationalisieren (Maxime 3 und 4).

Implizit nimmt Nisters aber genau den affectus-Aspekt auf, wenn er vom Kind spricht, das ,without any further thought“ das Geschenkt einfach annimmt und damit spielt. Aber zeigt es nicht gerade damit seine Dankbarkeit? Und noch mehr: wer nicht dankbar zu sein versteht, ist nicht nur im Unrecht gegenüber denen, denen er Dank schuldet, sondern er schadet am allermeisten sich selbst. Denn wie sollte jemand glücklich sein, wenn er nicht dankbar ist? Diese Verbindung ist es, die das spielende Kind versinnbildlicht.

3. Die Stufenordnung der Dankbarkeit sowie der passiven und aktiven Undankbarkeit kann einen verwirren. Seltsamerweise zählt Nisters die drei Stufen der passiven Undankbarkeit in Fußnote 4 in der von Thomas angegebenen Reihenfolge auf, kehrt sie aber in seinem Referat im Text um: für Thomas ist die erste (und harmloseste) Stufe der Undankbarkeit, dass es nicht zu einer Gegengabe kommt. Die dritte und schlimmste (gravissimus) besteht darin, dass die empfangene Wohltat 
gar nicht als solche anerkannt wird. Die Pointe dieser Reihung ist, dass Sichtbarkeit und Schwere der Verfehlung in einem umgekehrten Verhältnis zueinander stehen: der Wohltäter sieht sehr wohl, dass eine Gegengabe ausbleibt - das ist aber nicht so schlimm, denn immerhin hat der Beschenkte die Wohltat anerkannt und das durch seine Danksagung auch geäußert. Das Nichtanerkennen der Wohltat hingegen ist die schlimmste Form der (passiven) Undankbarkeit, und sie bleibt vermutlich sogar ihrem ,Täter“ verborgen.

Die passive Undankbarkeit wird immer schlimmer, je passiver der Undankbare sich zeigt, die aktive hingegen nimmt stufenweise mit wachsender Aktivität zu.

4. Die ,quid pro quo“-Struktur, die der gewöhnlichen Ansicht der Dankbarkeit inhäriert, soll, so das Abstract von Nisters, in einer reifen Sittlichkeit transzendiert werden. Nisters scheint damit sagen zu wollen, dass, bei Lichte betrachtet, Franz gar nicht so undankbar ist, wie es auf den ersten Blick scheint, bzw. dass ,granger" (Dankbarkeits-Ärger) eben auch eine Form von Dankbarkeit darstellt. Aber wir müssen das quid pro quo gar nicht transzendieren.

Nachdem wir im ersten Punkt die Verdoppelung der schenkenden Handlung, der Gabe sowie der beschenkten Person erwogen hatten, fassen wir eine vierte Verdoppelung ins Auge (sie ist nur ein erweiterter Aspekt der ersten): nämlich die des reichen jungen Mannes. Er ist nämlich zunächst Auslöser von Dankbarkeit, nach der Erfahrung des im Nu zerronnenen Geschenks aber Auslöser von aktiver Undankbarkeit: ja, er hat den armen Franz noch ärmer gemacht, da sein übereiltes Geschenk diesem schmerzlich vor Augen geführt hat, dass in seinen Händen sich sozusagen Gold in Pech verwandelt.

5. Schließlich ein Blick auf den Schenkenden: er löst ja die ambivalenten Gefühle aus, Dank und Undank. Aber hat er mit seiner großzügigen Gabe eigentlich Franz gemeint? Es war ja Zufall, dass er statt der gängigen Münze in der Eile (und weil es für ihn gleichgültig war) zu einem Goldstück griff. Insofern kann man sagen: mit Blick auf den ,effectus“ war Franz undankbar, mit Blick auf den ,affectus“ eigentlich nicht. Das seltsame Gemisch der Reaktionen löst sich also einigermaßen auf, wenn man mit Thomas berücksichtigt, dass die Vergeltung der Gabe eher die Gesinnung (affectus) als die Zuwendung (effectus) des Gebers zu berücksichtigen hat (vgl. STh II-II, q. 106, a. 5; Deutsche Thomas-Ausgabe, Bd. 20, S. 87).

Funding Open Access funding enabled and organized by Projekt DEAL.

Open Access Dieser Artikel wird unter der Creative Commons Namensnennung 4.0 International Lizenz veröffentlicht, welche die Nutzung, Vervielfältigung, Bearbeitung, Verbreitung und Wiedergabe in jeglichem Medium und Format erlaubt, sofern Sie den/die ursprünglichen Autor(en) und die Quelle ordnungsgemäß nennen, einen Link zur Creative Commons Lizenz beifügen und angeben, ob Änderungen vorgenommen wurden.

Die in diesem Artikel enthaltenen Bilder und sonstiges Drittmaterial unterliegen ebenfalls der genannten Creative Commons Lizenz, sofern sich aus der Abbildungslegende nichts anderes ergibt. Sofern das betreffende Material nicht unter der genannten Creative Commons Lizenz steht und die betreffende Handlung nicht nach gesetzlichen Vorschriften erlaubt ist, ist für die oben aufgeführten Weiterverwendungen des Materials die Einwilligung des jeweiligen Rechteinhabers einzuholen.

Weitere Details zur Lizenz entnehmen Sie bitte der Lizenzinformation auf http://creativecommons.org/ licenses/by/4.0/deed.de. 